\title{
Atypical femoral fractures are a separate entity, characterized by highly specific radiographic features. A comparison of 59 cases and 218 controls
}

\author{
Jörg Schilcher, Veronika Koeppen, Jonas Ranstam, Ralf Skripitz, \\ Karl Michaelsson and Per Aspenberg
}

\section{Linköping University Post Print}

N.B.: When citing this work, cite the original article.

Original Publication:

Jörg Schilcher, Veronika Koeppen, Jonas Ranstam, Ralf Skripitz, Karl Michaelsson and Per Aspenberg, Atypical femoral fractures are a separate entity, characterized by highly specific radiographic features. A comparison of 59 cases and 218 controls, 2013, Bone, (52), 1, 389392.

http://dx.doi.org/10.1016/j.bone.2012.10.016

Copyright: Elsevier http://www.elsevier.com/

Postprint available at: Linköping University Electronic Press http://urn.kb.se/resolve?urn=urn:nbn:se:liu:diva-88359 


\section{Atypical femoral fractures are a separate entity, characterized by highly specific radiographic features. A comparison of 59 cases and 218 controls.}

Jörg Schilcher ${ }^{\mathrm{a}}$, Veronika Koeppen ${ }^{\mathrm{a}+\mathrm{c}}$, Jonas Ranstam ${ }^{\mathrm{b}}$, Ralf Skripitz ${ }^{\mathrm{b}}$, Karl Michaëlsson $^{c}$ and Per Aspenberg ${ }^{a}$

${ }^{\text {a }}$ Department of Orthopedic Surgery, University Hospital Linköping and Department of Experimental and Clinical Medicine, Faculty of Health Science, Linköping University, Linköping, Sweden

${ }^{\mathrm{b}}$ Department of Clinical Sciences, Orthopaedics, Lund, Lund University, Sweden

${ }^{c}$ Department of Orthopedic Surgery, University Hospital Rostock, Rostock, Germany

${ }^{\mathrm{d}}$ Department of Surgical Sciences, Section of Orthopedics, Uppsala University, Uppsala, Sweden

Jörg Schilcher and Veronika Koeppen contributed equally

Corresponding author: Jörg Schilcher

Phone: +46 (0)10-103 4312

Fax: $+46(0) 10-1034305$

E-mail: jorg.schilcher@lio.se

Address:

Department of Orthopedic Surgery

University Hospital Linköping

Linköping University

S-581 85 Linköping, Sweden 


\begin{abstract}
Background: Estimations of the risk of bisphosphonate associated atypical femoral fractures vary between different population-based studies, from considerable to neglectable. A possible explanation for these discrepancies could be different definitions of atypical fractures. We aimed to identify specific radiographic fracture characteristics associated with bisphosphonate use.
\end{abstract}

Methods: In a previous nationwide study, 59 atypical and 218 ordinary fractures were diagnosed. The atypical fractures were defined by their stress-type fracture pattern. All fractures were now re-assessed by a physician in training, without information about bisphosphonate use. The fracture angle ( 0 - 180 degrees $)$ was measured. Presence of local lateral cortical thickening (a callus reaction), more than 2 fragments, or a medial spike was noted. The reader then made a judgment whether the fracture appeared as an atypical fracture based on the ASBMR criteria.

Results: Frequency distribution analysis of the fracture angle showed a distinct subgroup, comprising $25 \%$ of all 277 fractures, with a mean of 89 and SD of 10 degrees. 42 of 57 patients in this subgroup used bisphosphonates, whereas only 27 of 213 others did (specificity $0.93 ; 95 \%$ CI $0.88-0.96$ ). Presence of a callus reaction had also a high specificity for bisphosphonate use (0.96; $95 \%$ CI 0.92 to 0.98$)$. The ASBMR criteria had a lower specificity, increasing the number of atypical fractures without bisphosphonate use from 13 to 31 . This led to a decrease in age-adjusted relative risk associated with bisphosphonate use from 47 (95\% CI 26 to 87) to 19 (95\% CI 12 to 29$)$.

Interpretation: Stress fractures of the femoral shaft are a specific entity, which is easily diagnosed on radiographs and strongly related to bisphosphonate use.

Differences in diagnostic criteria may partially explain the large differences in relative risk between different population-based studies.

Keywords: atypical femoral fracture, bisphosphonate, stress fracture, osteoporosis 


\section{Introduction}

\section{1 .1}

Large discrepancies remain in reported incidences of bisphosphonate associated atypical femoral fractures. The reasons for these discrepancies are unclear.

\section{1 .2}

Atypical femoral fractures are rare and mainly found in the elderly. Orthopedic surgeons who first recognized the fracture pattern where struck by its appearance, resembling typical features of stress, or fatigue, fractures. Fatigue fractures are well known from mechanical engineering, as they occur with age and overload in many materials. In medicine, they typically occur in the lower extremity of athletes. High tensional forces along the longitudinal axis of long bones cause fracture lines running perpendicularly to these forces. Crack propagation and the surrounding healing processes lead to a distinct appearance on x-ray. Typically, there is a transverse fracture line in the cortical bone of the diaphysis together with a localized cortical hypertrophy (fracture callus).

\section{1 .3}

While mechanical overload causes stress fractures in athletes, the pathological mechanism of atypical fractures in the elderly, osteoporotic patient using bisphosphonates is less clear. A potential mechanism is deterioration of bone mechanical properties caused by suppression of bone turnover[1,2]. It has been shown that bisphosphonate treatment can lead to hypermineralization of bone, reduced heterogeneity of mineralization and changes in collagen composition [3-5]. These and other factors can cause stiffening of the bone, leading to accelerated accumulation of microdamage and ultimately stress fracture. A different explanation is based on the fact that bisphosphonates in the circulation will bind to fresh fracture surfaces. These bisphosphonates might inhibit targeted, osteoclast-mediated remodeling when new microcracks occur, thus allowing the crack to grow and cause a stress fracture. The hypothesis of inhibition of targeted remodeling is supported by the observation that the risk of atypical fractures decreases rapidly after cessation of bisphosphonate treatment. $[1,1]$ 


\section{1 .4}

In 2010, the American Society for Bone and Mineral Research (ASBMR) published a classification system defining major and minor features of atypical femur fractures in order to provide unified criteria for both clinicians and researchers[3,6]. All of these criteria are typical for stress fractures except for the generalized cortical thickening among the minor criteria. However, the ASBMR criteria are not precisely defined, and typical features of stress fractures can be found in both the major and minor categories. The fractures that are classified as atypical might therefore vary substantially depending on how the criteria are used. If also patients without the typical stress fracture pattern become included, the strength of the association with bisphosphonate use is likely to be reduced.

\subsection{5}

Recent epidemiological studies, where fractures were classified according to radiographic appearance, show remarkable differences regarding the association between bisphosphonate use and atypical fractures. In a nation-wide study, henceforth called the Swedish 2008 cohort, Schilcher et al. found a relative risk of 47 in the cohort analysis and in their case-control analysis an adjusted odds ratio (OR) for atypical fractures in bisphosphonate users of 33[1,7]. In contrast, Feldstein et al. in a large epidemiologic study in the US found an OR of only 2 [6,8]. In the Swedish 2008 cohort, $78 \%$ of the patients with atypical fractures used bisphosphonates, whereas in the Feldstein study they were $30 \%$. In a smaller study by Girgis et al.[7,9], 85\% of the patients with a radiographic fatigue fracture pattern had used a bisphosphonate. The Schilcher and Girgis studies used radiographic features of stress fractures to identify atypical fractures while the Feldstein study analyzed $75 \%$ of the radiographs using the ASBMR criteria. The question arises whether the use of different radiographic criteria can explain such large discrepancies.

\subsection{6}

Difficulties to identify atypical fractures depend largely on to what extent they form a specific entity. If they are a well-separated type of fractures, also blunt definitions will identify them. However, it has been speculated that they represent one end of a continuum of osteoporotic fracture appearances. 


\section{1 .7}

The primary aim of this analysis was to clarify if atypical fractures constitute a welldelineated separate entity. The most important feature of a stress fracture is the transverse orientation of the fracture line on the tension side of the bone. Therefore, we analyzed the distribution of the fracture angle in cases and controls in the Swedish 2008 cohort.

\section{1 .8}

The secondary aim was to evaluate the specificity and sensitivity of different components of the radiographic ASBMR criteria to identify bisphosphonate treatment.

\section{1 .9}

Thirdly, we evaluated differences in atypical fracture incidence when applying radiographic ASBMR criteria instead of the traditional orthopedic criteria for stress fractures that were used in the published analysis of the Swedish 2008 cohort.

\section{Patients and methods}

\subsection{1}

\section{Study population}

The previous publication of the Swedish 2008 cohort included a case-control analysis of all 59 women with atypical femoral fractures and the 263 women with other fractures of the femoral diaphysis. 45 of the 263 control cases were excluded in this study. The reasons for exclusion were retained implants that were not initially detected (26 patients) and that fractures had major rather than minor involvement of the trochanter (19 patients). The regional ethics committee approved the study, and allowed patient enrolment without consent being obtained.

\subsection{1}

\section{Measurements of individual radiological features}

All radiographic measurements and classifications were made by a physician in training, blinded for all background information including drug treatment and without experience in radiology or orthopedics, after a briefing by the senior authors. 


\subsection{2}

Digital DICOM files of plain X-rays of the pelvis, hip, femur and knee were imported into the database of SECTRA's IDS7 Workstation, Version 14.1.0.503 and 12.5.0.264. Measurements were performed in random order using the digital toolbox. Cases where measurement could not be performed due to insufficient radiographic material are listed in Appendix 1.

\subsection{3}

Fracture angles were preferably measured on the AP radiographs of the hip or femur and on the proximal fragment of the fracture. One line was drawn parallel to the lateral cortex of the femoral diaphysis and the other parallel to the fracture line, extending from the lateral cortex into the shaft, approximately one third of its width. Since fracture lines are two-dimensional projections of a three-dimensional body, and $\mathrm{x}$-ray beams rarely run parallel to the plane of the fracture, we measured angles on the best visible fracture line. Where cortical overlaying made measurement from the outer margin of the lateral cortex impossible, measurements were started from the most lateral aspect of the visible fracture line. In atypical fracture cases, the periosteal callus formation was ignored when drawing the reference line along the lateral cortex (Appendix 2). For measurement of reproducibility, 20 fractures previously defined as stress fractures, and 20 other fractures, were selected by use of a computer-generated list of random numbers and remeasured by another author.

\section{2 .4}

Fracture callus was defined as cortical apposition beyond the margin of the normal cortex on the periosteal or endosteal side of the lateral cortex.

\subsection{5}

Number of fragments was defined as two fragments or more fragments. A fragment was considered separate when the displacement was greater than $3 \mathrm{~mm}$.

\subsection{6}

A medial spike was defined as the medial end of the fracture extending from the proximal or distal fragment to form a sharp spike. It typically occurs in transverse or 
short oblique fractures when the fracture line abruptly changes from horizontal to a steep slope.

\subsection{1}

Reclassification according to ASBMR major criteria

All 277 fractures were reclassified according to the ASBMR criteria. Fractures were classified as atypical only when all major features were present, minor features were not compulsory. The term transverse or short oblique, which is a central feature of the criteria, was interpreted as an angle of less than 30 degrees from a line drawn perpendicularly to the lateral femoral cortex, according to the AO classification REF. This is consistent with angles between 60 and 120 degrees, when measured from the lateral cortex.

\subsection{1}

\section{Statistical Analysis}

Frequency distribution curves were estimated for fracture angles (range $0-180^{\circ}$ ) using the R program for statistical computing, version 2.14.1 together with the mixtool package (Young D, et al. Tools for analyzing finite mixture models. April 7, 2012), software for mixture analysis[1,8].This software allows estimating the probability density function of a variable using the kernel density estimation method.

\subsection{2}

For calculation of specificity and sensitivity, fracture angles were separated into two groups, based on the frequency distribution analysis (Figure 1). One group contained angles from 75 to 105 degrees, the remaining cases formed the other group.

\section{4 .3}

The specificity and sensitivity for each radiological finding to detect bisphosphonate treatment were calculated with $95 \%$ confidence intervals (CI). Specificity was calculated as (no bisph, neg finding) / (no bisph, pos finding + no bisph, neg finding). Sensitivity was calculated as (bisph, pos finding) / (bisph, pos finding + bisph, neg finding). 


\subsection{4}

We also calculated specificity and sensitivity to detect stress fractures, as they were identified in the previous publication, which was based on common understanding of stress fracture characteristics[7,9,10].

\subsection{5}

The relative risk for atypical femur fracture in bisphosphonate users versus non-users was calculated after the reclassification of the Sweden 2008 cohort according to the ASBMR criteria. Age-adjustment was performed as previously described [1,11].

\section{Results}

\section{1 .1}

\section{Distribution of fracture angle}

The fracture angle showed a trimodal distribution, with one peak roughly between 75 and 105 degrees, and two broader peaks between 15-45 and 125-165 degrees (Fig. 1). Fractures with angles between the three peaks were rare.

\section{1 .2}

The frequency distribution analysis confirmed the trimodal distribution and showed that the subgroup represented by the central peak comprised $25 \%$ of all 277 fractures and had a mean of 89 and SD of 10 degrees (Fig. 2).

\subsection{3}

The double measurement of fracture angles yielded a good correlation with exception of 3 cases where one examiner had measured a proximally and the other a distally directed fracture line (Appendix 3). The two examiners' allocation of fractures to angles within or outside 75 to 105 degrees yielded a disagreement in only 2 cases (Kappa-Value 0.9).

\section{2 .1}

Distribution of radiographic features 
The features medial spike, callus reaction and fracture angle 75 to 105 degrees showed considerable overlap (Appendix 4). 36 patients showed all 3 features. No patient showed a callus reaction without having both a spike and an angle between 75 and 105 degrees.

\subsection{1}

\section{Radiographic appearance and bisphosphonate use}

42 of 57 patients with fracture angles between 75 and 105 degrees used bisphosphonates, whereas only 27 of the 213 others did. Presence of a callus reaction had a high specificity for bisphosphonate use $(0.96 ; 95 \%$ CI 0.92 to 0.98 . Fracture angle from 75 to 105 degrees also had a high specificity $(0.93 ; 95 \%$ CI $0.88-0.96)$ to detect bisphosphonate treatment. Number of fragments had the lowest specificity of all features $(0.48 ; 95 \%$ CI 0.41 to 0.55$)$ (Table 1$)$.

\subsection{2}

Using previously defined stress fracture cases as a standard, we found the highest specificity for radiographic callus reaction $(1.00 ; 95 \%$ CI $0.98-1.00)$. Fracture angle also had very high specificity $(0.98 ; 95 \%$ CI 0.95 to 0.99$)$. This confirms that the above criteria were the ones used for identifying stress fractures in the original classification. The moderate sensitivity of these variables is less interesting, as it only means that many patients using bisphosphonates still may get an ordinary osteoporosis fracture.

\subsection{1}

\section{Reclassification according to ASBMR major criteria}

80 fractures were found to fulfill all major ASBMR criteria. Of these, 49 patients used bisphosphonates. Of the remaining 197 patients, 20 used bisphosphonates. Thus, $61 \%$ of the patients with atypical fractures used bisphosphonates, compared to $78 \%$ in the original classification REF. There was a considerable overlap between the fracture angle criterion (75 to 105) and the previously published classification, but not with the ASBMR criteria (Appendix 5). 
The previously published analysis showed an age-adjusted relative risk of atypical fracture among bisphosphonate users of 47 (95\% CI 26 to 87). By using the ASBMR criteria, this risk estimate was reduced to 19 (95\% CI 12 to 29$)$.

\section{Discussion}

\section{1 .1}

The results of this study show a very distinct subgroup of fracture angles around 90 degrees in a well-defined cohort of femoral fractures. This finding supports our hypothesis that atypical femoral stress fractures are a separate fracture entity with minor overlap with ordinary osteoporotic fractures.

\section{1 .2}

The analysis of radiographic features showed that signs of a stress fracture (transverse fracture angle, local callus reaction and medial spike) had a high specificity for bisphosphonate treatment. The reclassification according to the ASBMR criteria resulted in a decrease of the estimated relative risk from 47 to 19. This decrease was mainly caused by an increased number of non-users classified as having atypical fractures. This supports our hypothesis that ASBMR criteria are less specific for bisphosphonate use than our original classification REF.

\subsection{3}

To our knowledge this is the first time that detailed measurements of fracture angles were used to define atypical femoral fractures. According to the AO classification, a transverse fracture has an angle less than 30 degrees, i.e. 60 to 120 degrees. However, our frequency distribution analysis showed that $95 \%$ of all fractures of the distinct transverse subgroup lie between 69 and 109 degrees. A narrower range of 75 to 105 degrees will cover slightly less than $90 \%$ of the subgroup, and this range yielded a high specificity for bisphosphonate use.

\subsection{1}




\subsection{2}

If a variable with a lower specificity is chosen, such as a fracture angle between 60 and 120 degrees, the number of atypical fractures will be overestimated, so that the relation to bisphosphonate use will become attenuated.

\section{2 .3}

Rosenberg et al[11] analyzed sensitivity, specificity and accuracy of radiographic features in a case control analysis of 38 diaphyseal fractures (19 with bisphosphonate use). Their results are similar to ours, especially in terms of specificity. The specificity for fracture angle was lower than in our study, but their criteria did not define a transverse fracture in terms of degrees. Reanalyzing our data with angles ranging from 60 to 120 degrees yields a specificity of 0.87 (data not shown), which is strikingly similar to those of Rosenberg et al.

\subsection{1}

We found a lower incidence of bisphosphonate use among atypical fractures when defined by the ASBMR major criteria compared to radiographic signs of stress fractures. A comparison of two recent studies (Feldstein et al[6] and Dell et al[12]) shows a similar difference. Both these studies used large cohorts from different Kaiser Permanente hospital regions, to evaluate incidence rates of atypical fractures in bisphosphonate users. Feldstein et al defined cases by the ASBMR major and minor criteria while Dell et al. used radiographic signs of stress fractures: transverse fracture pattern, thickening callus reaction at the fracture site and location of the fracture in the femoral diaphysis. Dell et al observed that $90 \%$ of atypical fracture patients used bisphosphonates, while Feldstein reported only 30\%. The ASBMR major criteria may have a lower specificity, and in accordance, Feldstein et al found only a moderate agreement between orthopedic experts for any atypical feature (kappa value 0.62).

\subsection{1}

Our measurements are based on a well-defined study population from the Sweden 2008 cohort. A physician blinded for background information including bisphosphonate use performed all measurements. However, there are weaknesses: the results are limited to a female, Caucasian population from a Nordic country, and we have not evaluated the effect of age or treatment duration on the results. Measurement 
of fracture angles on a two-dimensional radiograph is problematic, as the fracture in reality has a complex three-dimensional geometry. Every fracture consists of two fracture lines projecting over each other on a plain radiograph, and the angle measured will be distorted depending on the angle between the fracture and the radiographic beam. However, because the lateral part of the stress fractures are restricted to one plane, almost perpendicular to the long axis of the bone, the errors due to overprojection of two fracture lines will be small.

\subsection{1}

With the current evidence at hand it seems that criteria based on typical features of stress fractures are more closely related to bisphosphonate use than the ASBMR major criteria. A revision of these criteria therefore seems warranted, a suggestion already made by Feldstein et al. We suggest that a fracture angle between 75 and 105 degrees, a local callus reaction and fracture location in the diaphysis should be used to define cases of atypical femoral fractures.

\subsection{1}

We conclude that atypical femoral fractures are a separate fracture entity with highly specific radiographic features, resembling those of stress fractures. If they are identified correctly, the relation to bisphosphonate use is very strong. 
Fig. 1. Fracture angle distribution in relation to bisphosphonate use. Fracture angles were grouped in 10-degree increments. Note the high incidence of bisphosphonate treatment in the fracture angle groups 75 to 105 degrees.

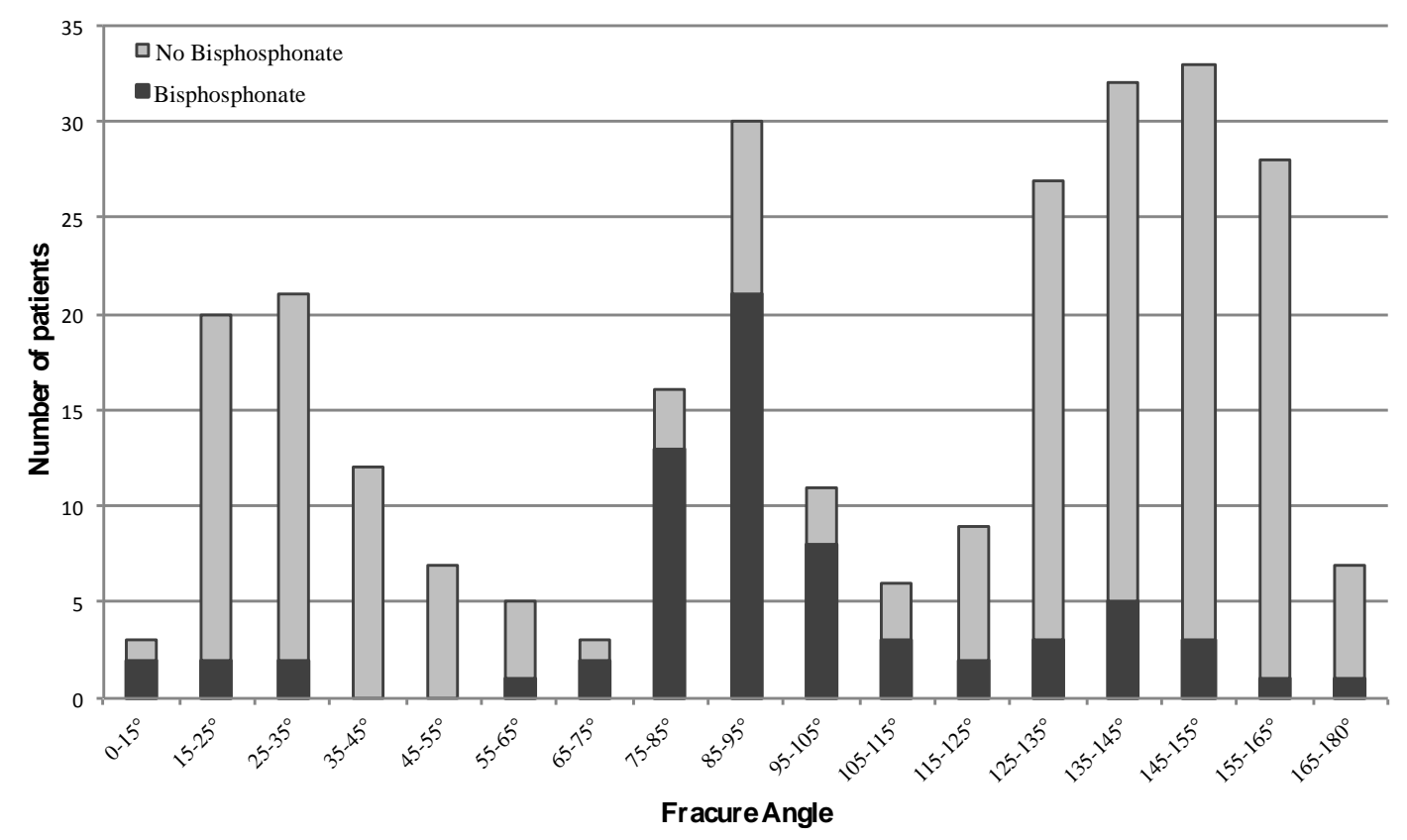


Fig. 2. Frequency distribution of fractures angles. Fracture angles occurred in three different peaks. The peak in the middle represents a distinct subpopulation with fracture lines running close to perpendicular to the lateral cortex of the femoral shaft, i.e. 89 SD 10 degrees.

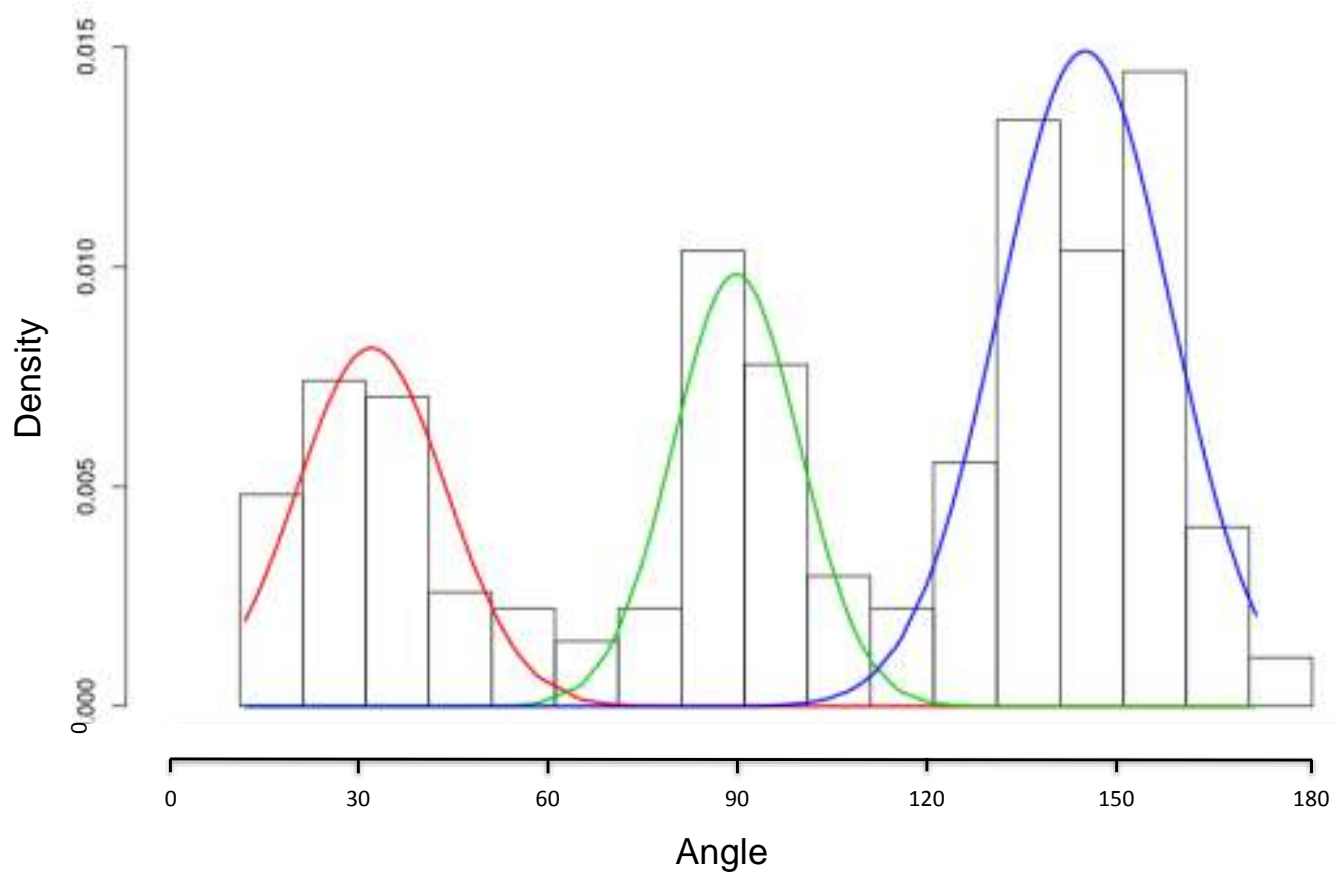


Table 1. Specificity and sensitivity of radiographic features to detect bisphosphonate treatment with $95 \%$ Confidence Intervals (CI).

Specificity $(95 \% \mathrm{CI}) \quad$ Sensitivity $(95 \% \mathrm{CI})$

Fracture angle $\quad 0.93(0.88-0.96) \quad 0.61(0.48-0.72)$

Callus $\quad 0.96(0.92-0.98) \quad 0.55(0.43-0.67)$

Medial Spike $\quad 0.93(0.88-0.96) \quad 0.54(0.41-0.66)$

Number of fragments $\quad 0.48(0.41-0.55) \quad 0.78(0.67-0.87)$ 
Appendix 1. Missing measurements for the different features in atypical cases and ordinary controls.

\begin{tabular}{|c|c|c|c|c|c|c|c|c|}
\hline \multirow[b]{2}{*}{$\begin{array}{c}\text { Patients } \\
n=277\end{array}$} & \multicolumn{2}{|c|}{ Angle } & \multicolumn{2}{|c|}{ Callus reaction } & \multicolumn{2}{|c|}{ Medial spike } & \multicolumn{2}{|c|}{ Number of fragments } \\
\hline & $\begin{array}{c}\text { measured } \\
n=270\end{array}$ & $\begin{array}{c}\text { not measured } \\
n=7\end{array}$ & $\begin{array}{c}\text { measured } \\
n=47\end{array}$ & $\begin{array}{c}\text { not measured } \\
n=230\end{array}$ & $\begin{array}{c}\text { measured } \\
\mathrm{n}=276\end{array}$ & $\begin{array}{c}\text { not measured } \\
\mathrm{n}=1\end{array}$ & $\begin{array}{c}\text { measured } \\
\mathrm{n}=276\end{array}$ & $\begin{array}{c}\text { not measured } \\
n=1\end{array}$ \\
\hline
\end{tabular}

\section{Atypical cases}

$\mathrm{n}=59$

Ordinary controls $\mathrm{n}=218$
59

211
0

59

217
7
217
59 
Appendix 2. Angle measurements in atypical and spiral fractures. Figure a: Atypical fracture. Measurements from the lateral margin of the lateral cortex. The reference line along the lateral cortex should ignore the beaking caused by the focal callus reaction. Figure b: Spiral fracture. Note the cortical overlaying at the lateral margin of the fracture line. Measurements started from the most lateral aspect of the visible fracture line.

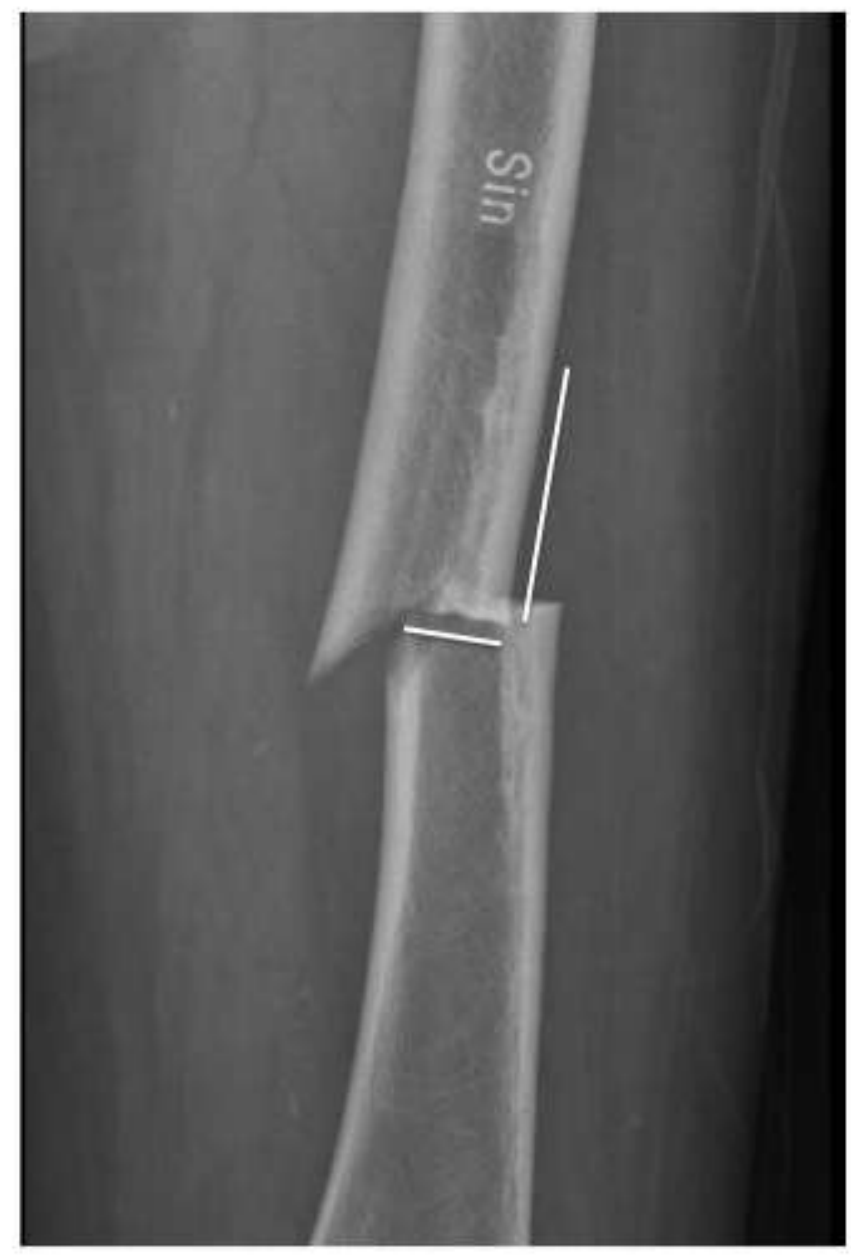

a

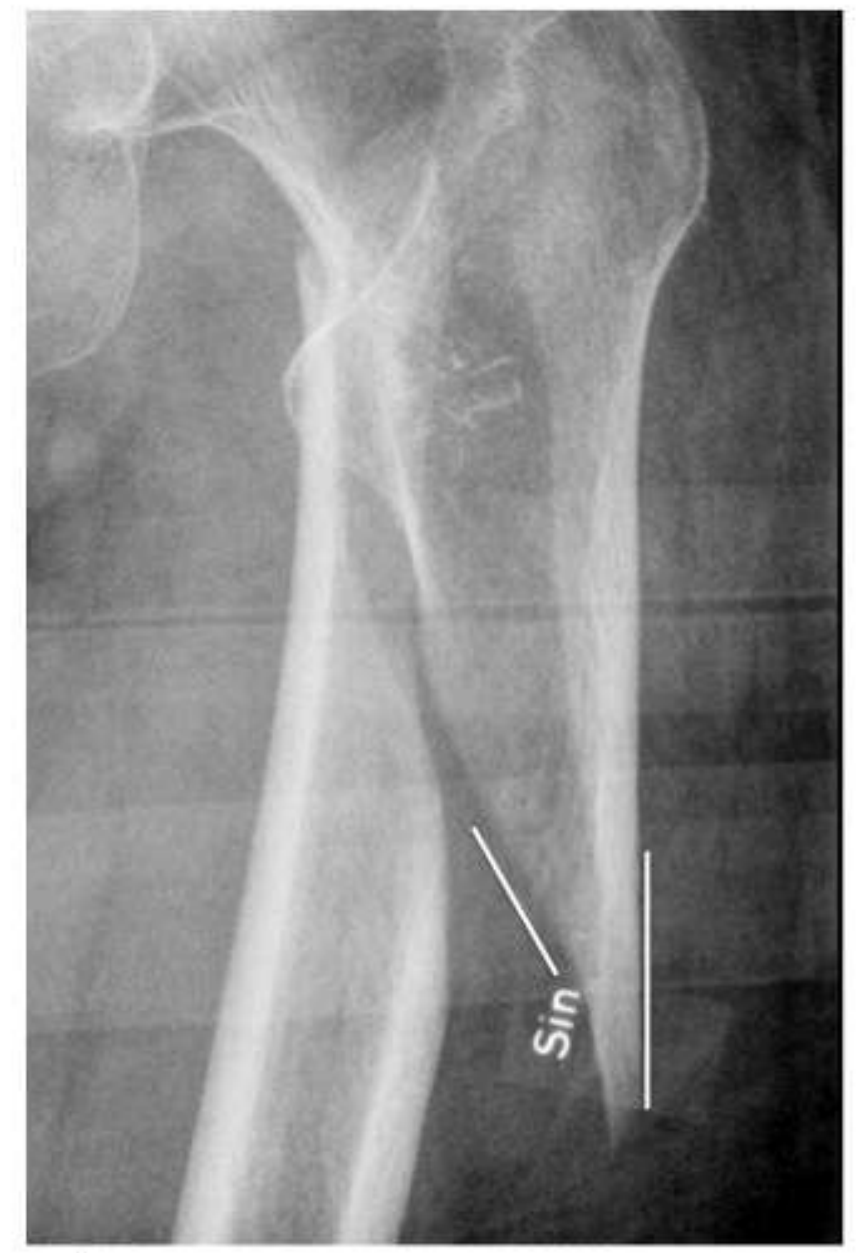

b 
Appendix 3. Double measurement of fracture angle by Examiner 1 and Examiner 2 for 20 randomly selected atypical fractures (according to previously published classification) and 20 controls. Three measurements with grossly differing values were caused by one examiner measuring a fracture line running proximally and the other examiner measuring one running distally.

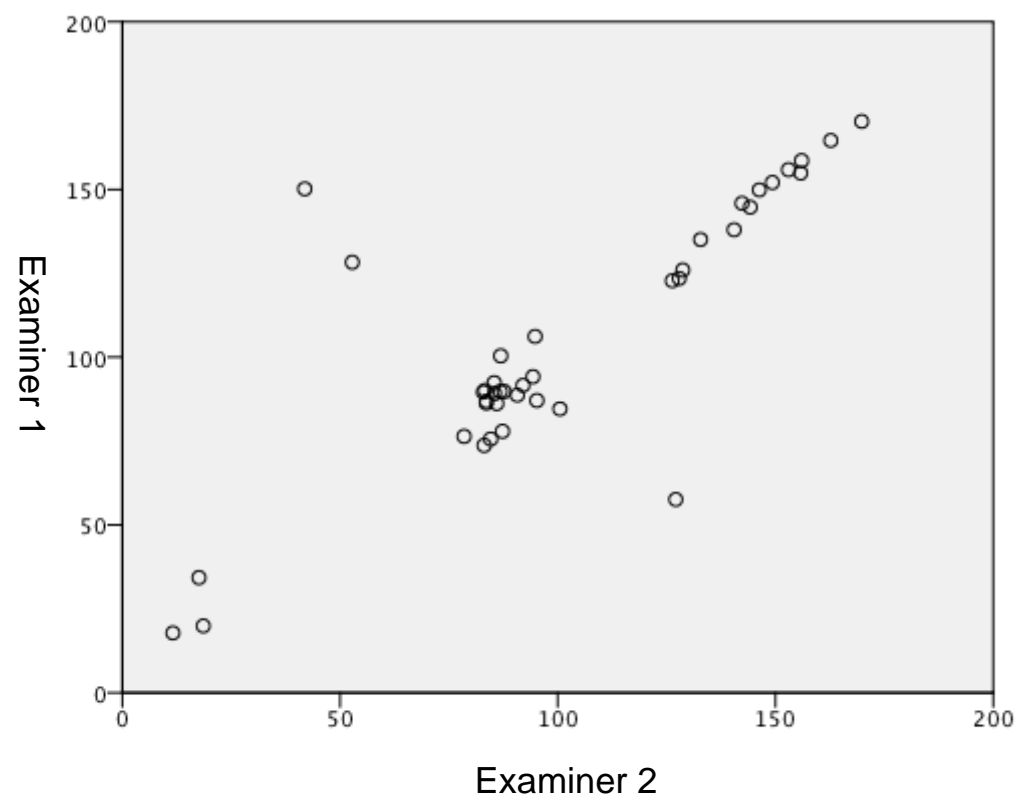


Appendix 4. Radiographic features of all atypical fractures and control fractures. The number of patients with bisphosphonate use are provided in brackets. Radiographic features overlapped for most patients. Callus reaction was always accompanied by at least one other radiographic feature, indicating high specificity. Fracture angle was defined as an angle between 75 and 105 degrees.

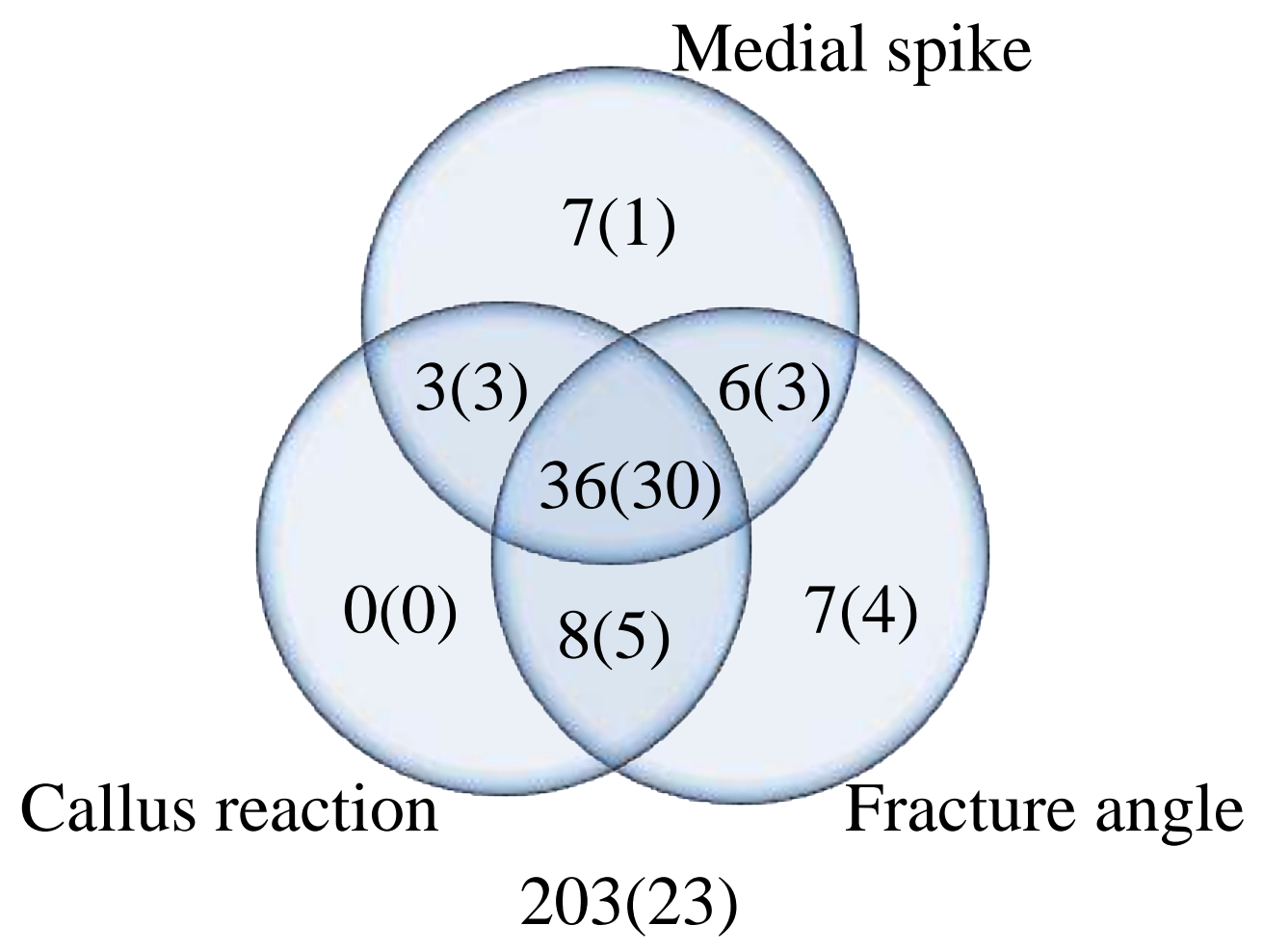


Appendix 5. Classification according to ASBMR criteria, original stress fracture classification and fracture angle ( 75 to 105 degrees). Of the 25 cases only fulfilling the ASBMR criteria, only 6 used a bisphosphonate.

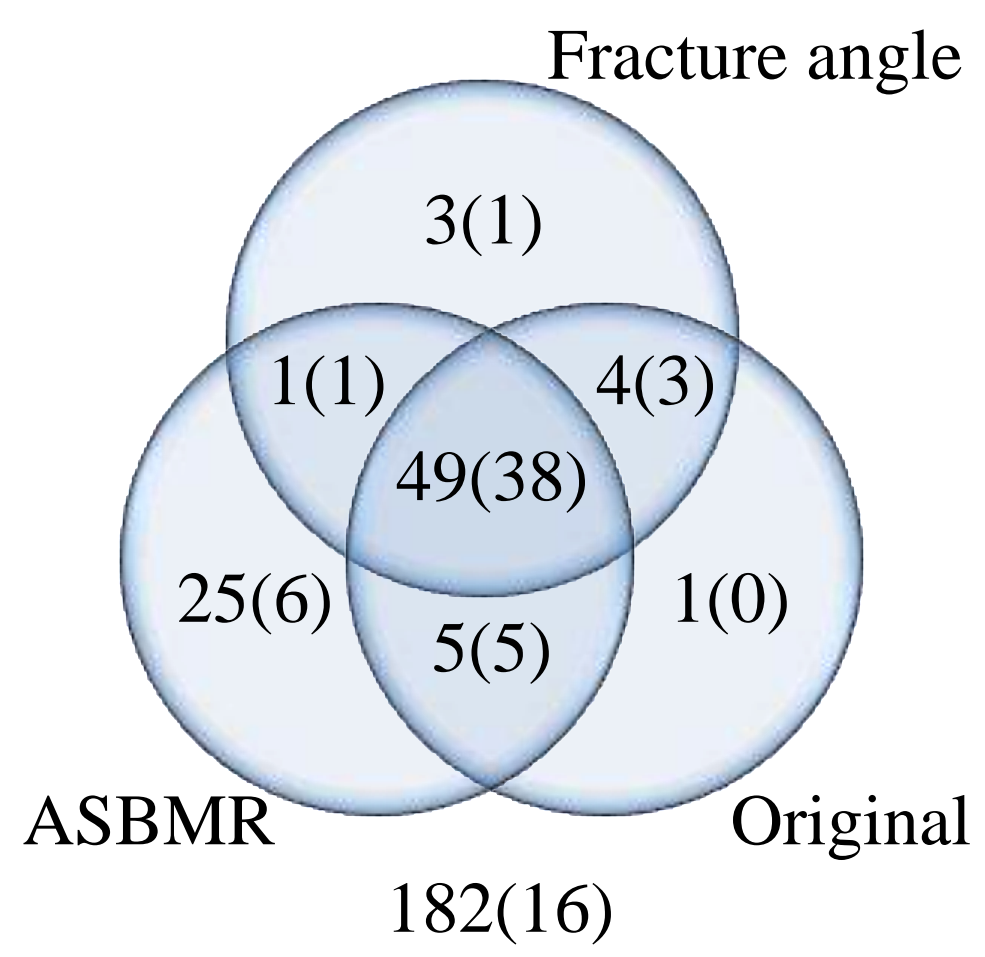


[1] Schilcher J, Michaëlsson K, Aspenberg P. Bisphosphonate use and atypical fractures of the femoral shaft. New England Journal of Medicine 2011;364:1728-1737.

[2] Tjhia CK, Odvina CV, Rao DS, Stover SM, Wang X, Fyhrie DP. Mechanical property and tissue mineral density differences among severely suppressed bone turnover (SSBT) patients, osteoporotic patients, and normal subjects. Bone 2011;49:1279-1289. doi:10.1016/j.bone.2011.09.042

[3] Shane E, Burr D, Ebeling PR, Abrahamsen B, Adler RA, Brown TD, et al. Atypical subtrochanteric and diaphyseal femoral fractures: Report of a task force of the american society for bone and mineral Research. J Bone Miner Res 2010;25:2267-2294. doi:10.1002/jbmr.253

[4] Donnelly E, Meredith DS, Nguyen JT, Gladnick BP, Rebolledo BJ, Shaffer $\mathrm{AD}$, et al. Reduced cortical bone compositional heterogeneity with bisphosphonate treatment in postmenopausal women with intertrochanteric and subtrochanteric fractures. J Bone Miner Res 2012;27:672-678.

[5] Tang SY, Allen MR, Phipps R, Burr DB, Vashishth D. Changes in nonenzymatic glycation and its association with altered mechanical properties following 1-year treatment with risedronate or alendronate. Osteoporos Int 2009;20:887-894. doi:10.1007/s00198-008-0754-4

[6] Feldstein A, Black D, Perrin N, Rosales AG, Friess D, Boardman D, et al. Incidence and demography of femur fractures with and without atypical features. J Bone Miner Res 2012.

[7] Girgis CM, Sher D, Seibel MJ. Atypical Femoral Fractures and Bisphosphonate Use. The New England journal of medicine 2010;13;362(19):1848-9.

[8] McLachlan G, Peel D. Finite Mixture Models. John Wiley \& Sons, Inc. 2000

[9] Moran DS, Evans RK, Hadad E. Imaging of lower extremity stress fracture injuries. Sports Medicine 2008;38:345-356.

[10] Lenart BA, Neviaser AS, Lyman S, Chang CC, Edobor-Osula F, Steele B, et al. Association of low-energy femoral fractures with prolonged bisphosphonate use: a case control study. Osteoporos Int 2008;20:1353-1362. doi:10.1007/s00198-008-0805-x

[11] Rosenberg ZS, La Rocca Vieira R, Chan SS, Babb J, Akyol Y, Rybak LD, et al. Bisphosphonate-Related Complete Atypical Subtrochanteric Femoral Fractures: Diagnostic Utility of Radiography. American Journal of Roentgenology 2011;197:954-960. doi:10.2214/AJR.10.6262

[12] Dell RM, Adams AL, Greene DF, Funahashi TT, Silverman SL, Eisemon $\mathrm{EO}$, et al. Incidence of atypical nontraumatic diaphyseal fractures of the femur. J Bone Miner Res 2012:n/a-n/a. doi:10.1002/jbmr.1719 